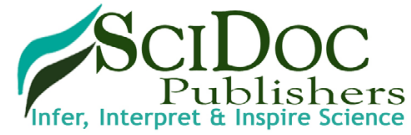

\section{Dapsone in the Treatment of Epidermolysis Bullosa Acquisita Long - Term Follow - Up}

Case Report

Elloudi S*, El Mahi H, Salim G, Mernissi FZ

Department of Dermatology and Venereology, University Hospital Hassan II Fez, Morocco.

\title{
Abstract
}

Epidermolysis bullosa acquisita (EBA) is a rare, acquired, chronic subepidermal bullous disease of the skin and mucosa characterized by autoantibodies to type VII collagen structures, a major component of anchoring fibrils, that attach the epidermis onto the dermis. The treatment of EBA is based on anecdotal reports in the literature with systemic therapies. We report a case of EBA successfully treated by dapsone.

Keywords: Epidermolysis Bullosa Acquisita; Dapsone.

\section{Introduction}

EBA is a rare, acquired, chronic subepidermal bullous disease of the skin and mucosa characterized by autoantibodies to type VII collagen structures, a major component of anchoring fibrils, that attach the epidermis onto the dermis. The treatment of EBA is based on anecdotal reports in the literature with systemic therapies. We report a case of EBA successfully treated by dapsone.

\section{Case Report}

A 54 years old, chronic smoker, admitted to our department for itching bullous dermatosis lasting for 2 weeks. Clinical examination revealed clear strained vesicular bubbles based on an erythematous skin, grouped like rosette, clear and hemorrhagic bubble flaccid and tense., sitting at the neck, forearms, elbows, hands, knees, feet, navel hives plates at the forearm, trunk, periorbital erosions, erosions of the hard palate, erosive cheilitis (Figures $1,2,3,4)$. Nikolski was negatf. The histology and immunofluorecence confirmed diagnosis of EBA, and the patient was treated with colchicine and oral steroids, without improvement, then, was treated with methotrexate without any improvement. Then, we opted for dapsone, with good wound healing with a decline of 2 years.

\section{Discussion}

EBA is a rare, autoimmune bullous disease with a prevalence of approximately 0.2 per million people and has an unknown etiology [1]. The classical presentation is a mechanobullous disease marked by skin fragility over trauma-prone surfaces. Causing a non-inflammatory bullous disease with an acral distribution that heals with scarring and milia formation. Other presentations of EBA have been reported: mucosal predominant appearance reminiscent of cicatricial pemphigoid, bullous pemphigoid- like presentation and IgA-EBA [2].

Making a definitive diagnosis of EBA could be difficult because specialized tests available in only some laboratories are necessary to confirm the clinical suspicion.

The histology shows dermal-epidermal separation at the basement membrane and minimal inflammation. Immunoelectron microscopy demonstrating immune deposits on anchoring fibrils and immunoblotting or enzyme-linked immunosorbent assay (Elisa) detecting autoantibodies recognizing the type VII collagen [3].

EBA frequently is associated with Crohn's disease and an inflammatory bowel disease must be ruled out in patients with EBA and abdominal manifestations.

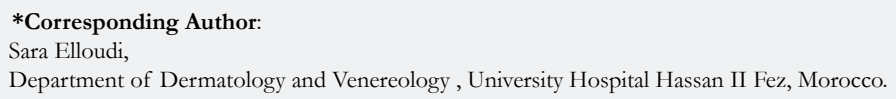

Copyright: Elloudi S 2016. This is an open-access article distributed under the terms of the Creative Commons Attribution License, which permits unrestricted use, distribution and reproduction in any medium, provided the original author and source are credited. 
Figure 1. Bubbles in the Neck and Buccal Mucosa.

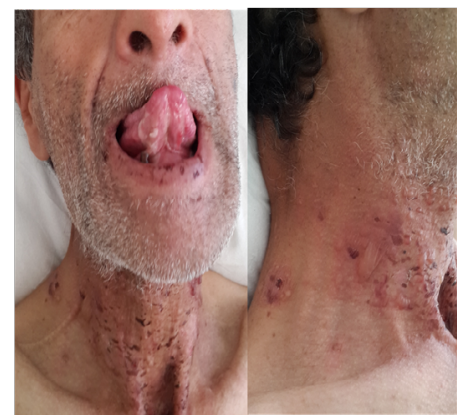

Figure 2. Bubbles and Erosions at the Area of Friction (axillary) and Trauma (elbow).

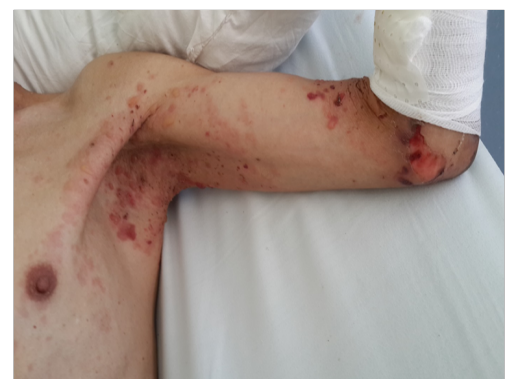

Figure 3. Bubbles and Erosions at the Area of Trauma (Hand and Feet).

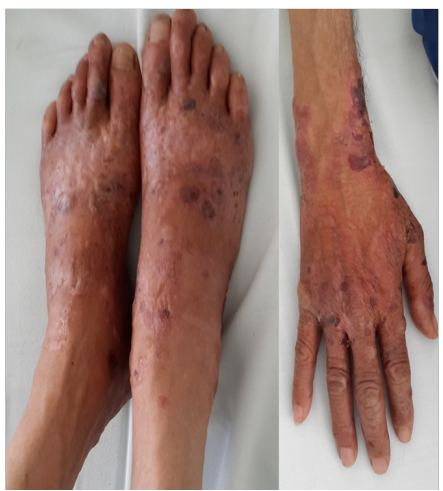

Figure 4. Cicatrisation of Erosions in Atrophic Mode.

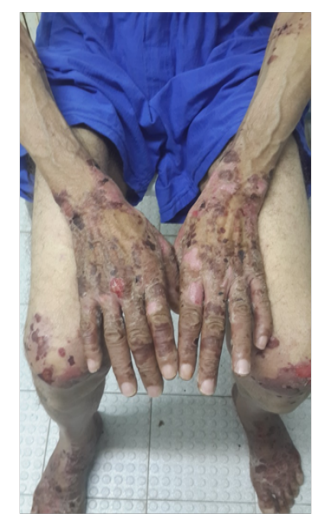

Therapy for EBA patients is unpredictable and often ineffective [4]. The therapy modality depends on the presentation of EBA. Supportive therapy is necessary for all EBA patients to help reduce the risk of complications and improve the quality of life. The first-line drug for EBA therapy is prednisone. Other immunosuppressants may also be tried including methotrexate, azathioprine, and cyclophosphamide. Dapsone and colchicine can help some EBA patients, especially those with neutrophils in their dermal infiltrate like our patient [5].

\section{Conclusion}

Because treatment choices are limited, dapsone could still be a viable alternative for patients with EBA who have contraindications to or failed other systemic therapies. Larger multicenter ran- 
domized controlled trials are needed to evaluate the efficacy and role of dapsone for the treatment of EBA.

\section{References}

[1]. Frédéric Caux (2010) Epidermolysis bullosa autoimmune. Autoimmune bullous diseases. 39(10): 1081-1088.

[2]. Vaillant L, Lorette G (1999) Bullous Autoimmune Diseases of the Oral Mucosa. Rev Stomatol Chir Maxillofac. 100(5): 230-9.
[3]. Prost C, Godeau P, Herson S, Piette JC (1996) Autoimmune bullous dermatosis of the dermo-epidermal junction. (3rd edn), Collection Medecinesciences Flammarion. 713-715.

[4]. Engineer L, Ahmed RA (2001) Emerging treatment for epidermolysis bullosa acquisita. J Am Acad Dermatol. 44(5): 818-28.

[5]. Prost-Squarcioni C, Ingen-Housz-Oro S, Joly P, Bernard P, Bedane C (2011) Epidermolysis bullosa acquisita. Guidelines for the diagnosis and treatment. Centres de reference des maladies bulleuses auto-immunes. Societe Francaise de Dermatologie. Ann dermatol vénéréol. 138(3): 274-279. 